\title{
Vigilância de óbitos infantis \\ em sistemas locais de saúde: avaliação da autópsia verbal e das informações de agentes de saúde ${ }^{1}$
}

\author{
Ivana C. H. C. Barrêto, ${ }^{2}$ Lígia Kerr Pontes ${ }^{2}$ e Luciano Corrêa ${ }^{3}$
}

RESUMO Conhecer os eventos vitais de uma população é de fundamental importância para combater a morbimortalidade e melhorar as condições de vida. Contudo, no Brasil, os sistemas de informação de saúde têm-se mostrado ineficientes. No presente estudo, realizado em três municípios do Estado do Ceará (Quixadá, Icapuí e Jucás), com boa cobertura de serviços de atenção primária à saúde, foram investigados, através de um instrumento epidemiológico denominado autópsia verbal, 215 óbitos de crianças menores de 1 ano de idade, representando 90\% do total dos óbitos em 1993 e 1994. Foram averiguadas as características socioeconômicas, cuidados e higiene, estado nutricional, o processo de doença, assistência e morte, a causa básica do óbito e o funcionamento do sistema de informação sobre mortalidade e do sistema de informação de agentes de saúde. Segundo a autópsia verbal, 39\% dos óbitos tiveram como causa básica a diarréia, seguida da prematuridade (17\%) e infecção respiratória aguda (10\%); 49\% das crianças morreram no domicílio, embora $79 \%$ das famílias tenham procurado os serviços de saúde no decorrer da doença fatal, sugerindo uma baixa efetividade na identificação e no tratamento de lactentes com doenças graves. Em $84 \%$ dos casos, a família procurou a rezadeira. Os agentes de saúde foram procurados em $29 \%$ dos casos, embora tenham notificado $78 \%$ dos óbitos investigados. A concordância estatística entre diagnóstico dos agentes de saúde para a causa básica do óbito e autópsia verbal foi boa para a diarréia, regular para outras causas, e fraca para a infecção respiratória aguda. A incorporação da autópsia verbal à rotina dos serviços de atenção primária à saúde do Estado propiciaria informações valiosas para as equipes locais de saúde e geraria uma consciência crítica que favorece a redução da mortalidade infantil.

\footnotetext{
1 Este estudo teve apoio financeiro do Projeto Nordeste, através da Secretaria Estadual de Saúde do Ceará, Escola de Saúde Pública do Estado do Ceará, Prefeitura Municipal de Quixadá, Prefeitura Municipal de Icapuí e Prefeitura Municipal de Jucás.

2 Secretaria de Saúde do Estado do Ceará e Secretaria Municipal de Saúde de Sobral. Correspondência e pedidos de separatas devem ser enviados a esta autora no seguinte endereço: Rua Noeme Dias Ibiapina 335, Bairro Junco, CEP 62030-320, Sobral, CE, Brasil. Fone/fax: +55-88614-1299. E-mail: ivana@sobral.ce.gov.br

3 Universidade Federal do Ceará, Departamento de Saúde Comunitária.
}

O conhecimento dos eventos vitais de uma população é de fundamental importância para o planejamento e execução hábeis de ações de saúde que visem combater a morbimortalidade e melhorar as condições de vida. No Brasil, com o atual processo de municipalização do sistema de saúde, estas informações devem ser coletadas e analisadas inicialmente em nível dos sistemas locais de saúde (SILOS), antes de serem encaminhadas para os níveis regional, estadual e central. Entretanto, os sistemas de informação de saúde
(SIS) no Brasil têm-se mostrado ineficientes. Às falhas que ocorrem no processo de notificação associa-se a centralização na consolidação dos dados, levando a um retardo considerável na análise das informações e, conseqüentemente, no planejamento e execução de ações para a melhoria das condições de saúde no âmbito local (1).

$\mathrm{O}$ registro oficial de eventos vitais, como nascimentos (declaração de nascidos vivos, DN) e óbitos (declarações de óbito, DO), segue o padrão acima mencionado. Em regiões pobres e com 
acesso difícil aos serviços de saúde, como o Nordeste do Brasil, estes registros tendem a ser escassos e de baixa qualidade $(2,3)$. É necessário, portanto, que se busquem soluções para este problema.

Em 1987, a primeira pesquisa sobre saúde materno-infantil no Estado do Ceará (PESMIC I, um estudo transversal com base populacional) revelou um sub-registro de $77 \%$ dos óbitos infantis em geral (4). Esta percentagem variou consideravelmente conforme a região: enquanto em Fortaleza mais da metade dos óbitos haviam sido registrados, no interior do Estado esta percentagem foi de cerca de $20 \%$. Na PESMIC III, estudo com metodologia similar à PESMIC I, realizado em 1994, a percentagem de registro de óbitos infantis no interior do Ceará foi de 30\% $(5,6)$. A subnotificação de óbitos infantis ocorre porque muitas crianças morrem em casa, pela dificuldade de acesso aos serviços oficiais de saúde, sendo enterradas em cemitérios clandestinos (7).

No Ceará, a partir de 1987, o governo estadual iniciou a implantação de um programa de agentes de saúde (PAS). Segundo a definição do programa, os agentes de saúde são pessoas que atuam na comunidade onde residem, realizando tarefas de prevenção e atenção primária à saúde; são remunerados pelo Estado com um salário mínimo ao mês e são capacitados e supervisados por técnicos da rede de saúde.

Todos os meses, os agentes de saúde notificam verbalmente ao seu supervisor os óbitos de crianças de 0 a 12 meses incompletos que ocorreram em sua área, classificando-os em três categorias: 1) óbitos por diarréia; 2) óbitos por infecção respiratória aguda; e 3) óbitos por outras causas. Estas informações devem ser consolidadas localmente e devem embasar o planejamento das secretarias municipais de saúde. Também devem ser encaminhadas mensalmente à coordenação estadual do PAS.

Este subsistema de informação contínuo e baseado na comunidade tem sido de grande utilidade para acompanhar a situação de saúde no Ceará, produzindo indicadores como taxas de mortalidade infantil, de mortalidade infantil por diarréia e por infecção respiratória aguda e de mortalidade proporcional para menores de 1 ano. Antes da implantação do sistema, estes indicadores só podiam ser obtidos por estudos epidemiológicos transversais, tendo em vista os elevados índices de sub-registro do sistema oficial de informação.

Por outro lado, faz-se necessário averiguar a qualidade das informações produzidas pelos agentes de saúde. Esta avaliação torna-se ainda mais importante se considerarmos que no Ceará, desde 1993, todos os 184 municípios do interior do Estado estão sendo atendidos com o PAS e que a cobertura média de famílias foi de $77 \%$ no período de 1993 e $1994^{4}$ e de $78 \%$ em 1995 e 1996. O número de agentes de saúde contratados pelo Estado do Ceará até dezembro de 1996 totalizou 8 353. O Ministério da Saúde, com base em resultados positivos de uma pesquisa sobre o seu programa de agentes comunitários de saúde (PACS), realizada em 1994 em 12 municípios de oito estados da região Nordeste (excetuando o Ceará, que possui um programa específico) também está desenvolvendo esforços para expandir o programa para os municípios mais pobres do país (8).

O programa de saúde da família (PSF), criado em 1994 pelo Ministério a partir de experiências piloto como a do município de Quixadá (9), propõe-se realizar uma prática sanitária que incorpora à atenção médica tradicional uma lógica de promoção à saúde. A idéia do PSF é constituir uma equipe de saúde composta por médico, enfermeira e auxiliares de enfermagem que trabalhariam em conjunto com os agentes de saúde de um determinado território-população, com o objetivo de prestar atenção integral à saúde das famílias da área (10). O PACS e o PSF estão sendo adotados como projetos prioritários do Ministério da Saúde no Brasil.

\footnotetext{
4 UNICEF Fortaleza. Infant mortality: recent tendencies in the Northeast of Brazil and State of Ceará (1992/94). Fortaleza: 1994. [Documento mimeografado]
}

Dentro deste contexto surgiu a idéia de montar uma estratégia para investigação dos óbitos infantis no âmbito local, a partir dos óbitos identificados pelos agentes de saúde e também através da pesquisa em cartórios e hospitais e planejar ações para redução da mortalidade, a partir dos dados obtidos. Como boa parte dos óbitos ocorrem nos domicílios, sem assistência médica, seria necessária a utilização de um instrumento que fosse capaz de identificar as características e circunstâncias dos óbitos através do testemunho da mãe ou de outro familiar das crianças falecidas.

Considerou-se, então, que a autópsia verbal, instrumento epidemiológico que tem sido amplamente utilizado em países em desenvolvimento para determinar causas de óbito por meio de entrevistas com parentes de crianças falecidas que não estavam sob supervisão médica na ocasião do falecimento (11), poderia ser uma alternativa para investigar óbitos que deixaram de ser registrados pelo sistema oficial de informação no Ceará.

A autópsia verbal se originou de uma proposta feita nos anos 50, quando Biraud elaborou um instrumento simplificado para investigar óbitos, com o objetivo de realizar diagnósticos comunitários (11). Depois de 20 anos, a Organização Mundial da Saúde (OMS) aperfeiçoou este instrumento, que se baseia em entrevistas com parentes da pessoa falecida para chegar à doença que mais provavelmente foi a causa básica do óbito (10). Em alguns estudos, os questionários têm perguntas abertas e perguntas estruturadas, com seqüências de questões (algoritmos) que predizem o diagnóstico médico e excluem outros diagnósticos. A técnica de autópsia verbal foi utilizada em estudos descritivos (12-14) e em estudos controlados com intervenções dirigidas a doenças específicas (15).

Nas Filipinas, Kalter et al. (16), com o objetivo de determinar a validade desta metodologia e definir a sensibilidade e especificidade de algoritmos diagnósticos, compararam sintomas e sinais relatados pelas mães utilizando um questionário estruturado, com 
diagnósticos médicos selecionados, para 164 óbitos de crianças com menos de 2 anos. A técnica teve $100 \%$ de sensibilidade para o tétano neonatal e $98 \%$ para o sarampo. A especificidade para o sarampo foi de $90 \%$. Para infecções respiratórias agudas (IRA), o algoritmo com tosse prolongada e dispnéia teve uma sensibilidade baixa, de $41 \%$, e $93 \%$ de especificidade. Para diarréia, o diagnóstico baseado em evacuações freqüentes e perda de líquidos nas fezes teve alta sensibilidade (78 a $84 \%$ ) e especificidade $(79 \%)$, a despeito de a criança ter falecido apenas com diarréia ou por diarréia associada a outra doença (15).

No Quênia, Snow et al. (10) realizaram um estudo prospectivo de 303 óbitos infantis no qual a técnica de autópsia verbal foi avaliada por comparação com os diagnósticos médicos confirmados disponíveis; a especificidade para detectar causas comuns de óbito foi superior a 80\% (10). Martínez et al. (17) destacaram que a autópsia verbal é uma interface entre a epidemiologia e a etnografia e representa uma ferramenta útil para investigar e dirigir estratégias para diminuir a mortalidade infantil (17).

O presente estudo originou-se do sistema de vigilância de nascimentos e óbitos infantis implementado entre dezembro de 1993 e julho de 1995 nos municípios cearenses de Icapuí, Jucás e Quixadá em parceria com as secretarias municipais de saúde e Secretaria Estadual de Saúde do Ceará com financiamento do Projeto Nordeste. Neste projeto original, utilizou-se a técnica de autópsia verbal com o objetivo principal de traçar o perfil das mortes de crianças menores de 5 anos e implementar um modelo de vigilância para os óbitos que pudesse ser mantido como rotina pelas secretarias municipais de saúde, constituindo-se num projeto de pesquisa-intervenção.

O presente relato refere-se à descrição, conforme autópsia verbal, informação dos agentes de saúde e DO, das características e circunstâncias dos óbitos de crianças menores de 1 ano ocorridos nos municípios de Icapuí, Jucás e Quixadá em um período de 2 anos. Além disso, o estudo propõe es- tratégias adequadas para a redução da mortalidade neste grupo etário e faz uma avaliação da adequação das três formas de identificação e caracterização de óbitos infantis aos sistemas municipais de saúde no Ceará.

\section{MATERIAIS E MÉTODOS}

O delineamento do estudo foi transversal com base populacional. Foram selecionados três municípios: Quixadá, município de médio porte, localizado no Sertão Central; Icapuí, município de pequeno porte localizado no litoral leste; e Jucás, município também de pequeno porte, situado na região centro-sul do Estado do Ceará.

Segundo o censo da Fundação Instituto Brasileiro de Geografia e Estatística (IBGE), em 1991 os três municípios apresentavam situação semelhante à média dos demais municípios cearenses: população predominantemente jovem, níveis precários de saneamento básico, elevadas taxas de analfabetismo e baixa renda familiar. Quixadá diferenciava-se dos outros dois municípios selecionados por apresentar, além de maior número de habitantes, uma concentração populacional maior na zona urbana (18-21). Por outro lado, estes municípios se caracterizavam, também, por ter serviços de saúde municipalizados e, no período de realização do estudo, por ter boa cobertura do PAS (implantado no mínimo há 4 anos) e por estar organizando seus sistemas locais de saúde com ênfase na atenção primária.

A população do estudo foi constituída de todas as crianças menores de 1 ano que morreram de janeiro de 1993 a dezembro de 1994 nestes municípios. Foram identificados 237 óbitos. Os óbitos de crianças com idade entre 0 e 11 meses e 29 dias foram identificados por meio da notificação dos agentes de saúde e pela investigação semanal nos livros dos cartórios de registro civil e nos hospitais municipais.

Para investigação em profundidade das características dos óbitos, utilizouse um questionário adaptado de um modelo de autópsia verbal utilizado pela Organização Pan-Americana de
Saúde (OPAS), aplicado de janeiro de 1994 a julho de 1995 (22). O questionário foi utilizado para pesquisar circunstâncias do nascimento, cuidados com a criança, situação social da família e características e circunstâncias do processo de doença, assistência e morte de cada criança.

As famílias das crianças receberam explicações sobre os objetivos do estudo, tendo sido solicitada sua autorização para aplicação da autópsia verbal. Foram entrevistadas, preferencialmente, as mães das crianças e, em segundo lugar, outro adulto que participasse no cuidado da mesma, geralmente a avó. Os agentes de saúde da área acompanharam os entrevistadores facilitando a interlocução com as famílias. Não houve recusa para responder ao questionário. Do total de 237 óbitos de menores de 1 ano, 22 (9\%) não foram investigados, ou porque a família da criança havia mudado de endereço e não pôde ser localizada, ou por falta de transporte para os entrevistadores. Em um caso, na zona rural de Quixadá, o óbito foi conseqüência de filicídio por espancamento, não sendo possível entrevistar os pais. Dessa forma, 215 mortes foram investigadas.

A obtenção do número de nascidos vivos foi efetuada em Jucás e Icapuí a partir das informações do PAS. Nesses municípios, a cobertura do PAS era próxima a 100\%. Em Quixadá, onde a cobertura do PAS era menor, a informação foi obtida junto ao subsistema de notificação de nascidos vivos (SINASC/MS), que registrava os nascimentos na maternidade local e em casas de parto (pequenas unidades de saúde onde as gestantes são atendidas por parteiras), assim como a informação dos agentes de saúde, no caso de partos domiciliares.

As causas básicas de óbito, segundo a autópsia verbal, foram tabuladas por faixa etária, sexo, período do ano, região onde morava a criança e município e classificadas em quatro grupos: 1) causas perinatais; 2) causas infecciosas; 3) causas externas e não infecciosas; e 4) causas não definidas.

Para fins do presente estudo, a desnutrição foi considerada como causa básica nos eventos em que a causa ini- 
cialmente determinada pela revisão da autópsia verbal havia sido diarréia, infecção respiratória aguda ou outra infecção e em que havia desnutrição grave associada. Para cálculo do grau de desnutrição utilizou-se o peso registrado no mês em que ocorreu o óbito. Foram classificados como graves os casos em que havia desnutrição de III grau segundo o critério de Gómez (23). O grau de desnutrição só foi calculado nos casos em que havia registro do peso no cartão da criança durante a entrevista.

As informações das DO que haviam sido preenchidas para os casos participantes do estudo foram comparadas com as da autópsia verbal. Foram também colecionadas todas as notificações de óbito de menores de 1 ano pelos agentes de saúde, abrangendo o mesmo período do estudo, com as seguintes informações: nome do agente de saúde, data de ocorrência do óbito, endereço da criança e causa básica do óbito atribuída pelo agente de saúde. Estas informações também foram comparadas com as informações da autópsia verbal.

Em cada município foi selecionado um profissional local de nível superior, enfermeira ou assistente social, para supervisar a investigação dos óbitos. A coordenadora técnica do estudo, médica pediatra e infectologista, reuniu-se com os supervisores locais para discussão do instrumento de autópsia verbal. Os entrevistadores foram ou os próprios supervisores ou estudantes de Medicina do $6^{\circ}$ ano, treinados pela coordenadora do estudo ou pela supervisora local. Cada autópsia verbal foi revisada pelo supervisor municipal em conjunto com o entrevistador responsável. Em casos de respostas inconsistentes ou duvidosas, a família foi novamente visitada para esclarecimento. Após revisão da autópsia verbal pela supervisora local, a coordenadora técnica do estudo analisou todas as entrevistas realizadas, determinando ao final qual a causa básica do óbito em questão, considerando as informações da autópsia verbal. Os casos para os quais não foi possível determinar a causa básica de óbito por meio das informações obtidas pela au- tópsia verbal, porque as informações eram vagas ou insuficientes, foram designados como tendo causa básica não definida.

Em vista da inexistência de DO para $123(57 \%)$ dos óbitos infantis investigados, as causas básicas dos óbitos determinadas pela revisão da autópsia verbal foram consideradas como padrão ouro; este padrão foi utilizado na validação das informações sobre causas de óbito fornecidas pelos agentes de saúde. Nesta análise foram considerados três diagnósticos básicos, para cuja identificação os agentes de saúde foram treinados: diarréia, IRA e causas distintas de diarréia e IRA.

Foram utilizadas três medidas de associação para analisar a notificação de óbito dos agentes de saúde com relação às causas básicas determinadas pelas autópsia verbal: sensibilidade, especificidade e índice Kappa, que mede a concordância estatística interobservadores, variando de +1 (concordância total) a -1 (discordância total) (24). O Kappa foi utilizado para avaliar a concordância entre a causa básica registrada na DO e aquela determinada pela autópsia verbal e entre a causa básica determinada pela autópsia verbal e aquela informada pelos agentes de saúde.

Os programas utilizados foram o Fox, para alimentação dos dados, e o Epi Info 6.1, para geração de tabelas e análise dos resultados.

$\mathrm{Na}$ descrição dos resultados, as informações sobre as crianças menores de 1 ano que foram a óbito em Quixadá, Icapuí e Jucás no período do estudo foram comparadas com as informações de crianças cearenses menores de 3 anos obtidas pela PESMIC III. As causas básicas de óbito das crianças menores de 1 ano nos municípios do estudo foram comparadas com as causas básicas dos óbito notificados através do sistema de informações sobre mortalidade (SIM) do Ministério da Saúde em 1994 para crianças cearenses menores de 1 ano (25).

\section{RESULTADOS}

O intervalo entre a ocorrência dos óbitos e a autópsia verbal variou de 1 a 13 meses. Em $45 \%$ dos casos, este intervalo foi de 1 a 6 meses e em $52 \%$, de 7 a 13 meses. Na tabela 1 pode-se observar que foram identificados um total de 237 óbitos pelos diversos meios empregados; destes, 215 (90\%) foram investigados por autópsia verbal.

A proporção de crianças com baixo peso ao nascer entre os municípios do estudo variou de 15 a $30 \%$, enquanto que, segundo dados do SINASC, no Ceará esta proporção foi de $8 \%$ no mesmo período (figura 1). Houve uma proporção maior de óbitos em crianças do sexo masculino tanto nos municípios do estudo $(P<0,01)$ como no Estado do Ceará em geral.

A duração da amamentação foi menor entre as crianças participantes do estudo em relação às crianças cea-

TABELA 1. Formas de identificação dos óbitos investigados com autópsia verbal, Quixadá, Jucás e Icapuí (CE), Brasil, 1993 e 1994

\begin{tabular}{lcccr}
\hline & \multicolumn{4}{c}{$\begin{array}{c}\text { Óbitos identificados em crianças } \\
\text { menores de 1 ano }\end{array}$} \\
\cline { 2 - 5 } \multicolumn{1}{c}{ Fontes de identificação } & Quixadá & Icapuí & Jucás & Total \\
\hline Agente de saúde, autópsia verbal e & 24 & 11 & 7 & 42 \\
$\quad$ declaração de óbito & 77 & 13 & 37 & 127 \\
Agente de saúde e autópsia verbal & - & - & 1 & 1 \\
Agente de saúde e declaração de óbito & 16 & - & - & 16 \\
Autópsia verbal e declaração de óbito & 1 & 8 & 10 & 19 \\
Agente de saúde & 24 & 1 & 5 & 30 \\
Autópsia verbal & 2 & - & - & 2 \\
Declaração de óbito & 144 & 33 & 60 & 237 \\
$\quad$ Total & & & & \\
\hline
\end{tabular}


FIGURA 1. Características das crianças do Estado do Ceará e dos menores de 1 ano que foram a óbito em Quixadá, Icapuí e Jucás (CE), Brasil, 1993 e 1994

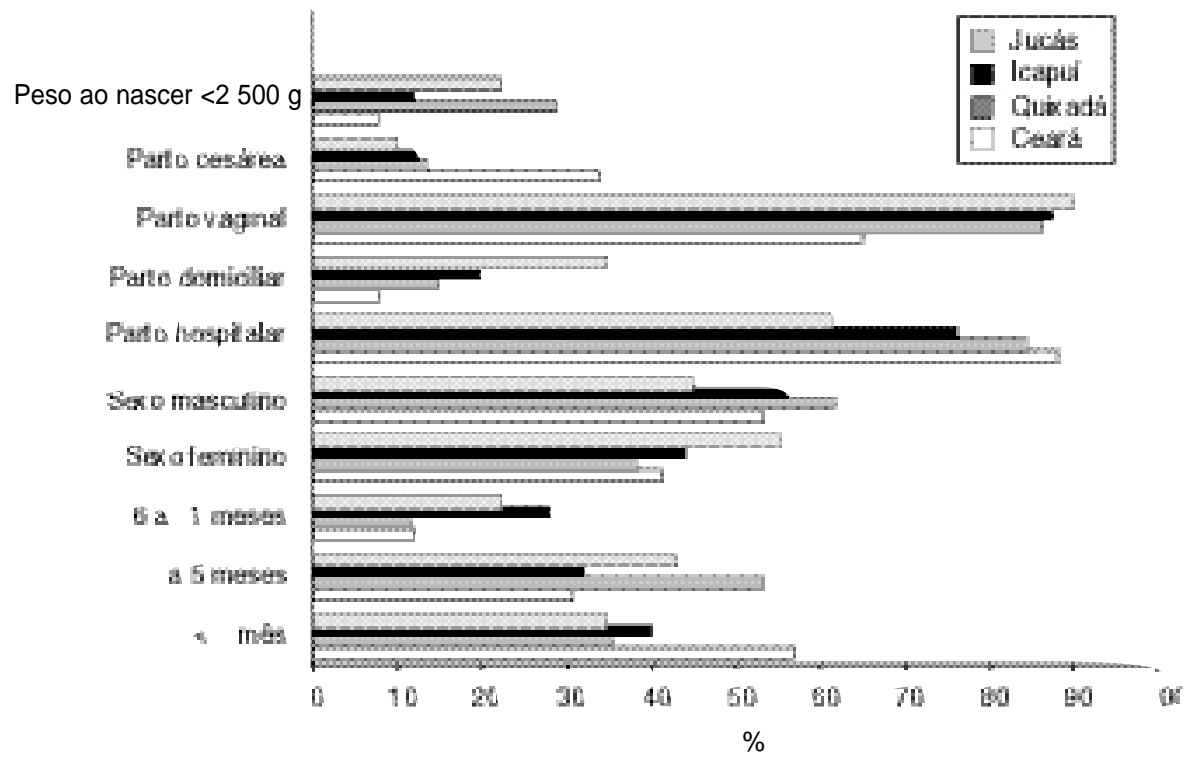

Fonte: Terceira pesquisa sobre saúde materno-infantil no Estado do Ceará (PESMIC III), sistema de informação sobre mortalidade (SIM) do Ministério da Saúde e autópsias verbais.

renses menores de 1 ano conforme a PESMIC III. Apenas 34\% do primeiro grupo mamaram durante os 60 primeiros dias de vida, contra $86 \%$ do segundo grupo (figura 2).

As condições socioeconômicas, avaliadas pelo tipo de moradia, presença de banheiro e possessão de eletrodomésticos, mostraram-se bem inferiores entre as crianças que foram a óbito nos municípios do estudo com relação às crianças cearenses menores de 3 anos, do interior do Estado, investigadas na PESMIC III. Apenas 8\% das famílias das crianças deste estudo possuíam geladeira, contra 29\% das famílias do interior do Ceará (figura 3).

A tabela 2 mostra os coeficientes de mortalidade infantil (CMI) nos três municípios do estudo em comparação com o CMI para o Estado do Ceará. É importante destacar que a cobertura do SIM no Ceará em 1994 era de 63\%, o que pode justificar porquê o CMI para o Ceará, calculado com base nos dados do SIM e do SINASC, foi bem inferior ao calculado pelos dados do SIPAS, que naquele ano já estava funcionando em $94 \%$ dos municípios Cearenses. de menores de 1 ano no Estado do Ceará em 1994.

Entre os 215 óbitos investigados com autópsia verbal, $24 \%$ estavam associados a desnutrição de primeiro ou segundo graus. Em 21\% dos casos não havia desnutrição associada. Para 55\% das crianças não foi possível averiguar a existência de desnutrição pela ausência do registro do peso no cartão da criança.

Comparando-se as causas básicas de óbito registradas na DO com as causas de óbito segundo a revisão da autópsia verbal, sendo o primeiro documento considerado como padrão ouro, já que foi preenchido pelo médico que prestou assistência à criança durante a doença que levou ao óbito, observouse que, nos casos em que havia DO preenchida para o óbito, a concordância entre DO e autópsia verbal foi boa (tabelas 3 e 4 ).

Os óbitos infantis estudados com autópsia verbal ocorreram principalmente no período de janeiro a abril (meses mais quentes e chuvosos), no qual concentraram-se $40 \%$ dos casos. Houve maior freqüência de óbitos infantis na zona rural (57\%, contra $43 \%$ na zona urbana).

A proporção de óbitos hospitalares e domiciliares foi semelhante para o total dos casos estudados (48\% contra $46 \%$, respectivamente). Houve variações quando o local do óbito para causas básicas específicas foi estudado. Dos óbitos por diarréia, 59\% ocorre-

FIGURA 2. Estudo comparativo dos indicadores da utilização do sistema sanitário e da duração da amamentação entre crianças cearenses e menores de 1 ano que foram a óbito nos municípios de Quixadá, Icapuí e Jucás (CE), Brasil, 1993 e 1994

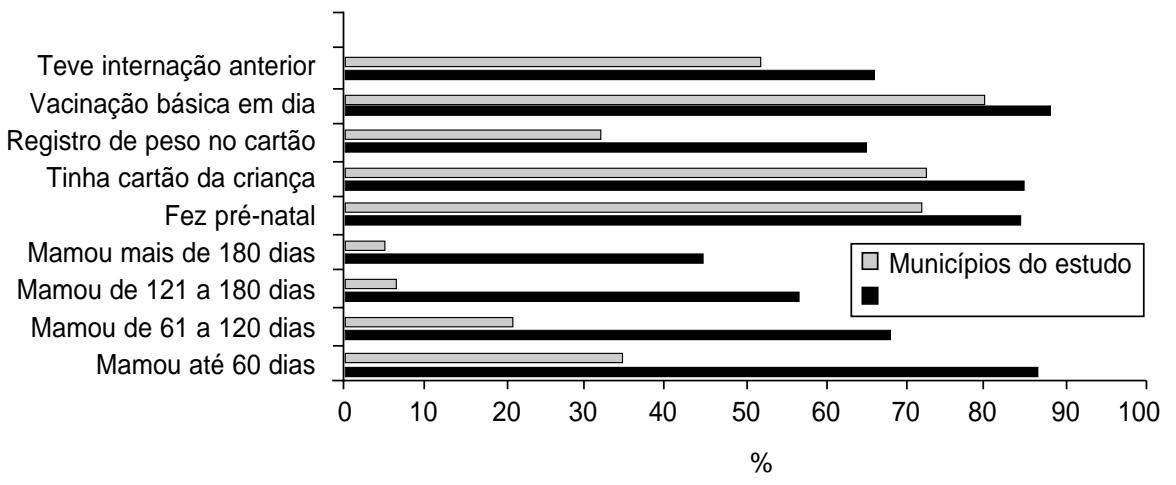

Fonte: Terceira pesquisa sobre saúde materno-infantil no Estado do Ceará (PESMIC III) e autópsias verbais. 
FIGURA 3. Indicadores socioeconômicos das crianças cearenses menores de 3 anos em 1994 e das crianças menores de 1 ano que foram a óbito nos municípios de Quixadá, Icapuí e Jucás (CE), Brasil, 1993 e 1994

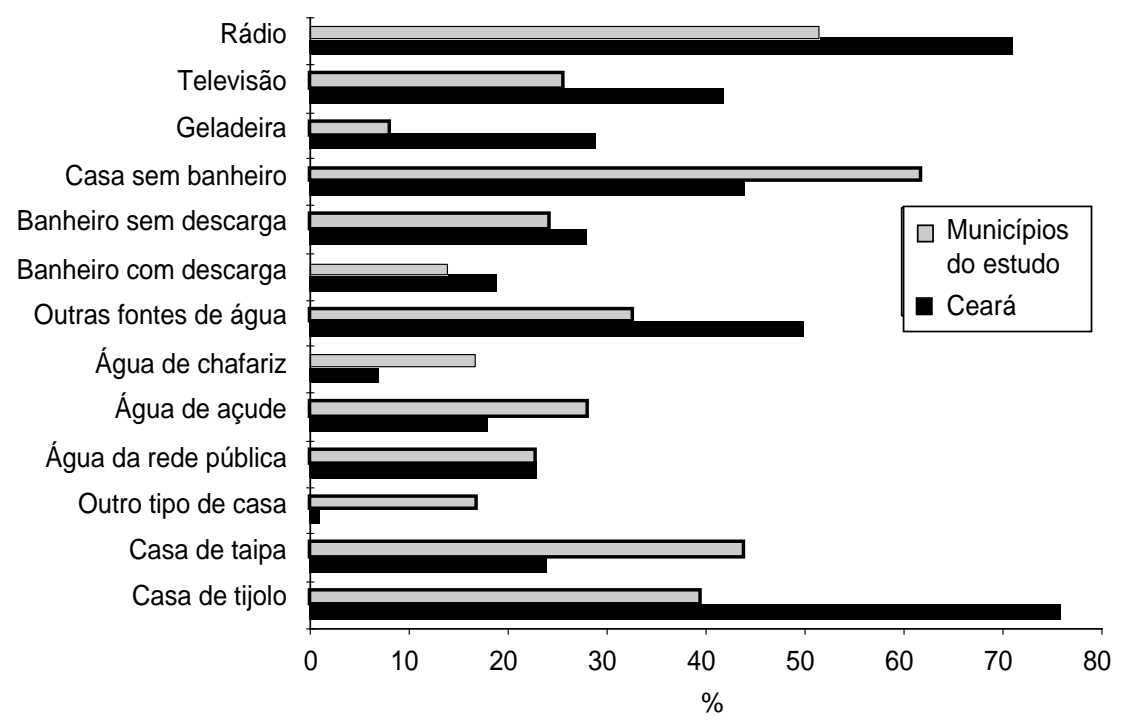

Fonte: Terceira pesquisa sobre saúde materno-infantil no Estado do Ceará (PESMIC III) e autópsias verbais. ram em casa. Já os óbitos por IRA e os óbitos por prematuridade ocorreram em sua maioria no hospital $(73 \%$ e $65 \%$, respectivamente).

Foram obtidas informações sobre o itinerário terapêutico de 207 crianças de 207 famílias. Setenta e nove por cento das famílias procuraram os serviços de saúde oficiais em algum momento da doença que ocasionou o óbito. Dentre as 163 crianças de famílias que procura- ram os serviços de saúde, $55 \%$ faleceram no hospital, 37\% em casa e 5\% em outro local. Foram levadas para a rezadeira, em algum momento da doença que ocasionou o óbito, 181 crianças. Em $13 \%$ dos casos, só a rezadeira foi procurada. Destes últimos casos, $65 \%$ tiveram diarréia como causa básica do óbito. Do total de 84 casos de óbitos por diarréia, $59 \%$ foram levados em algum momento da doença para a rezadeira. Os agentes
TABELA 2. Coeficientes de mortalidade infantil e mortalidade proporcional por causas no Estado do Ceará e nos municípios de Quixadá, Jucás e Icapuí (CE), Brasil, 1993 e 1994

\begin{tabular}{|c|c|c|c|c|c|}
\hline $\begin{array}{c}\text { Coeficientes de } \\
\text { mortalidade }\end{array}$ & $\begin{array}{l}\text { Ceará, } \\
1994^{b}\end{array}$ & $\begin{array}{l}\text { Ceará, } \\
\text { 1994c }\end{array}$ & $\begin{array}{c}\text { Quixadá, } \\
1993 \text { e } 1994\end{array}$ & $\begin{array}{c}\text { Icapuí, } \\
1993 \text { e } 1994\end{array}$ & $\begin{array}{c}\text { Jucás, } \\
1993 \text { e } 1994\end{array}$ \\
\hline Infantil ${ }^{d}$ & 56,2 & 80 & 37,9 & 43,9 & 59,8 \\
\hline Neonatale $^{\mathrm{e}}$ & - & - & 12,8 & 13,3 & 16,9 \\
\hline Pós-neonatale & - & - & 24,15 & 19,9 & 31,9 \\
\hline Causas perinatais $^{e}$ & 12,4 & - & 10,2 & 8,0 & 6,0 \\
\hline Causas infecciosas ${ }^{e}$ & 12,8 & - & 20,5 & 17,3 & 27,9 \\
\hline Causas não infecciosas ${ }^{e}$ & 8,18 & - & 2,3 & 1,3 & 9,0 \\
\hline Causas não definidas ${ }^{e}$ & 22,7 & - & 3,9 & 6,6 & 5,9 \\
\hline
\end{tabular}

a Por 1000 nascidos vivos.

b Segundo o sistema de informações sobre mortalidade do Ministério da Saúde.

c Segundo o subsistema de informação dos agentes de saúde.

d Calculado com base no total de óbitos identificados neste estudo (237 óbitos).

e Calculado com base no número total de óbitos investigados com autópsia verbal (215 óbitos) no caso dos municípios de Quixadá, Jucás e Icapuí. de saúde foram procurados em algum momento da doença por $29 \%$ das 207 famílias, apesar de terem notificado $78 \%$ dos óbitos estudados. Nos quatro casos em que a família referiu que não tomou nenhuma atitude a criança faleceu no domicílio.

As tabelas 4 e 5 comparam a informação obtida dos agentes de saúde à causa básica de óbito segundo a revisão da autópsia verbal.

\section{DISCUSSÃO}

O perfil de mortalidade encontrado nos três municípios do estudo, onde a diarréia foi a principal causa dos óbitos, seguida pela prematuridade e pela infecção respiratória aguda, é compatível com o esperado para regiões onde há elevada incidência de óbitos infantis com queda recente em conseqüência da penetração de cuidados primários de saúde. Ocorreu uma redução no número total de óbitos, principalmente resultante da redução das mortes após o período neonatal. No entanto, a diarréia persistiu sendo a principal causa, quase sempre associada à desnutrição. Este perfil de mortalidade é muito semelhante ao descrito por outros autores para duas cidades do interior do Nordeste em 1990 e 1991 (26).

Quanto à idade no momento do óbito, observa-se que, nos municípios do estudo, os óbitos ocorreram em sua maioria no período pós-neonatal, enquanto que, segundo dados do SIM para o resto do Estado do Ceará, em 1994 quase $60 \%$ dos óbitos ocorreram em crianças menores de 1 mês. Entretanto, vale lembrar o significativo subregistro do SIM, que chegou a $48 \%$ na nossa casuística, o que poderia explicar a diferença encontrada. Estas informações reforçam os dois graves problemas já discutidos anteriormente com relação ao SIM, o sub-registro e a ausência da definição correta da causa básica em uma grande percentagem de óbitos. O sub-registro do SIM devese, em parte, ao fato de que, como vimos na casuística de Quixadá, Icapuí e Jucás, quase metade dos óbitos infantis em geral e dois terços dos óbitos in- 
FIGURA 4. Causas básicas de óbitos infantis no Estado do Ceará em 1994 e nos municípios de Quixadá, Icapuí e Jucás (CE), Brasil, 1993 e 1994

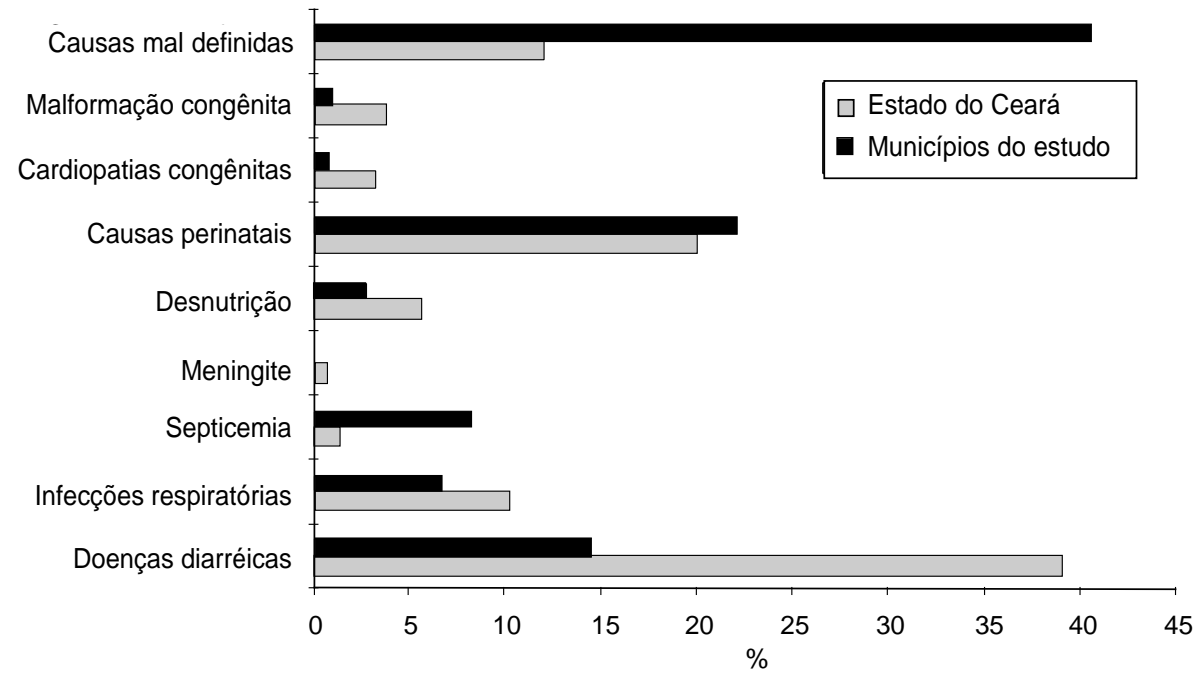

Fonte: Sistema de informação sobre mortalidade (SIM) do Ministério da Saúde e autópsias verbais.

fantis por diarréia ocorreram em casa e, portanto, não tinham DO.

A duração da amamentação entre as crianças participantes deste estudo foi bem menor que do que a registrada pela PESMIC III para o conjunto das crianças cearenses menores de 1 ano. Uma percentagem significativa da po- pulação infantil estudada correspondia a crianças que nunca foram amamentadas ou que mamaram por menos de 60 dias, o que pode ter contribuído para o grande número de óbitos no $1^{\circ}$ semestre de vida, em sua maioria por diarréia.

Apesar de a maioria das crianças falecidas possuírem o cartão da criança, em menos de um terço destes havia registro regular do peso. No relatório da PESMIC III, McAuliffe et al. (5) observaram que $65 \%$ das crianças estudadas tinham peso registrado no cartão nos últimos 3 meses e 33\% tinham peso recente registrado no cartão. Isto faz supor que o grupo de menores de 1 ano que faleceu pode corresponder a um grupo com menor cobertura de ações primárias de saúde com relação às demais crianças da mesma faixa etária nos três municípios. Isto alerta para que as equipes de saúde trabalhem no sentido de identificar na população de sua área de abrangência aquelas crianças que são menos assíduas nas unidades, o que pode ser realizado através dos agentes de saúde.

TABELA 3. Causas básicas de óbitos infantis segundo a declaração de óbito e a autópsia verbal, Quixadá, Jucás e Icapuí (CE), Brasil, 1993 e 1994

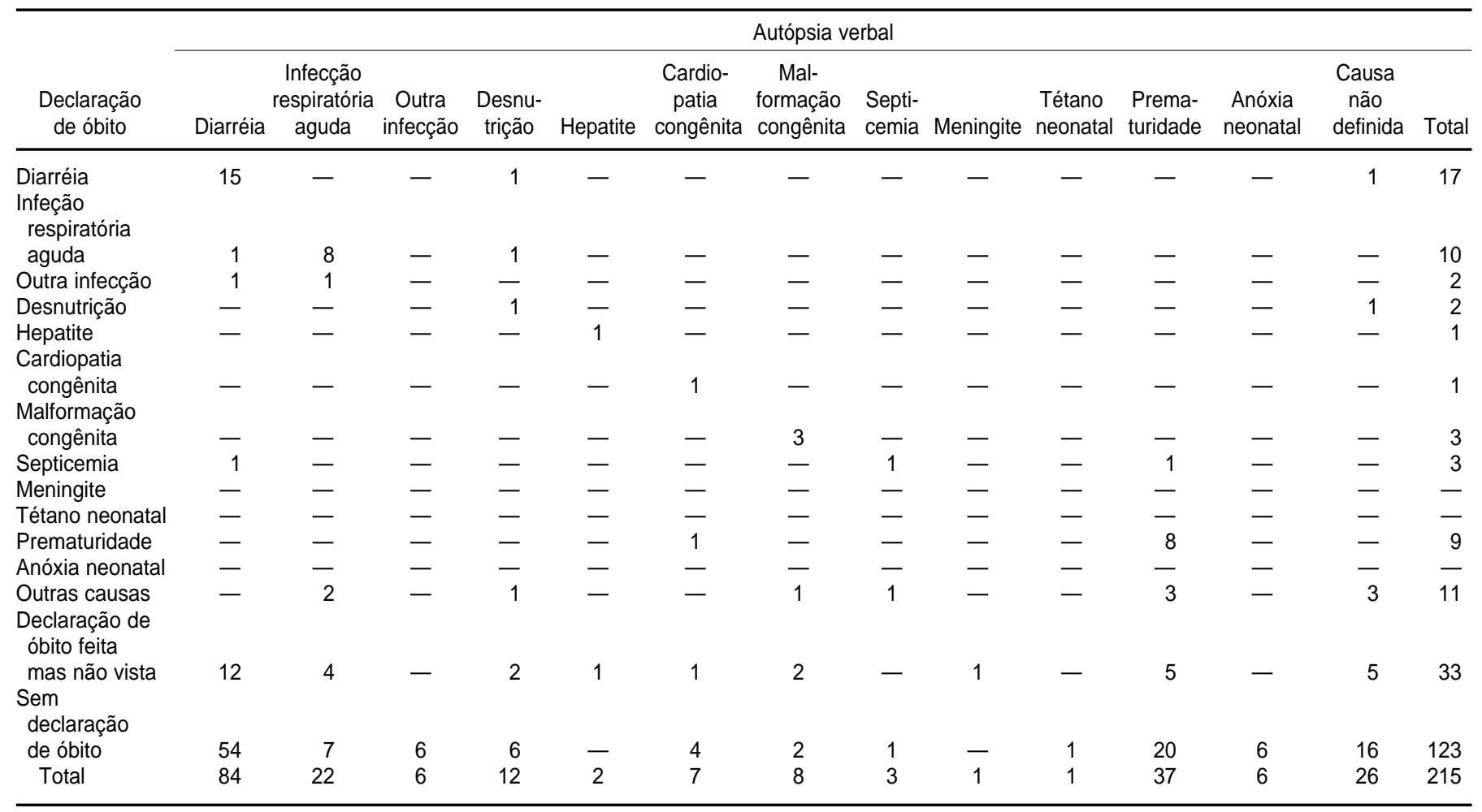


TABELA 4. Associação entre as causas básicas de óbito de menores de 1 ano registradas pela declaração de óbito, autópsia verbal e agentes de saúde, Quixadá, Jucás e Icapuí (CE), Brasil, 1993 e 1994

\begin{tabular}{|c|c|c|c|c|c|c|}
\hline \multirow[b]{3}{*}{ Causas básicas } & \multicolumn{6}{|c|}{ Medidas de associação } \\
\hline & \multicolumn{2}{|c|}{ Sensibilidade } & \multicolumn{2}{|c|}{ Especificidade } & \multirow{2}{*}{\multicolumn{2}{|c|}{ Kappa $^{\mathrm{a}}$}} \\
\hline & $\%$ & $\begin{array}{c}\text { Intervalo de } \\
\text { confiança }\end{array}$ & $\%$ & $\begin{array}{c}\text { Intervalo de } \\
\text { confiança }\end{array}$ & & \\
\hline \multicolumn{7}{|l|}{ Declaraccão de óbitob } \\
\hline Diarréia & 88 & $62-95$ & 93 & $79-98$ & 0,80 & Bom \\
\hline Infeccão respiratória aquda & 80 & $44-97$ & 94 & $82-98$ & 0.71 & Bom \\
\hline Prematuridade & 89 & $51-99$ & 92 & $80-97$ & 0,71 & Bom \\
\hline \multicolumn{7}{|l|}{ Agente de saúde } \\
\hline Diarréia & 64 & $51-75$ & 98 & $90-99$ & 0,64 & Bom \\
\hline Infecção respiratória aguda & 50 & $24-76$ & 90 & $83-94$ & 0,31 & Sofrível \\
\hline Outras causas & 82 & $72-89$ & 67 & $55-77$ & 0,49 & Regular \\
\hline
\end{tabular}

a $P<0,001$ para todos os coeficientes.

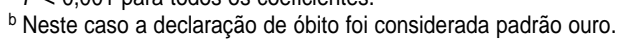

Em geral, o perfil socioeconômico encontrado foi compatível com o de famílias de muito baixa renda, inferior ao das crianças cearenses menores 3 anos participantes da PESMIC III, corroborando inúmeros estudos anteriores que relacionam a mortalidade infantil à situação socioeconômica desfavorável (27). A baixa freqüência de famílias que possuíam eletrodo- mésticos entre os casos estudados denota seu pobre acesso a bens de consumo. Não existia nem um rádio em metade dos domicílios e menos de um décimo das famílias possuíam geladeira. Quando forem planejadas ações educativas para redução da mortalidade infantil, outros recursos, além dos meios de comunicação de massa, devem ser utilizados.

TABELA 5. Causa básica de óbito segundo autópsia verbal nos casos em que o agente de saúde informou diarréia, infecção respiratória aguda e outras causas como causa do óbito, Quixadá, Icapuí e Jucás (CE), Brasil, 1993 e 1994

\begin{tabular}{|c|c|c|c|c|c|}
\hline \multirow[b]{2}{*}{ Autópsia verbal } & \multicolumn{5}{|c|}{ Causa básica segundo informação do agente de saúde } \\
\hline & Diarréia & $\begin{array}{c}\text { Infecção } \\
\text { respiratória } \\
\text { aguda }\end{array}$ & $\begin{array}{l}\text { Outras } \\
\text { causas }\end{array}$ & $\begin{array}{c}\text { Não } \\
\text { notificados }\end{array}$ & Total \\
\hline Diarréia & 41 & 4 & 19 & 20 & 84 \\
\hline Desnutrição & 2 & 1 & 4 & 5 & 12 \\
\hline Hepatite & 1 & 1 & - & - & 2 \\
\hline Infecção respiratória aguda & - & 7 & 4 & 11 & 22 \\
\hline Outra infecção & - & 1 & 4 & 1 & 6 \\
\hline Cardiopatia congênita & - & 2 & 3 & 2 & 7 \\
\hline Prematuridade & - & 5 & 18 & 14 & 37 \\
\hline Anóxia neonatal & - & 1 & 2 & 3 & 6 \\
\hline Malformações congênitas & - & - & 5 & 3 & 8 \\
\hline Septicemia & - & - & 3 & - & 3 \\
\hline Meningite & - & - & 1 & - & 1 \\
\hline Tétano neonatal & - & - & 1 & - & 1 \\
\hline Causa não definida & 1 & 1 & 11 & 13 & 26 \\
\hline
\end{tabular}

Mesmo nos três municípios estudados, onde já existiam serviços de atenção primária na zona rural, a mortalidade infantil foi mais elevada no meio rural. Em outros municípios do Estado, com menor cobertura dos agentes de saúde e com serviços de atenção primária menos acessíveis, provavelmente a concentração de óbitos na zona rural seja ainda maior. A estratégia do PSF, mais uma vez, mostra-se coerente com a realidade epidemiológica quando propõe a cobertura das áreas rurais com equipes de médicos, enfermeiros, auxiliares de enfermagem e agentes de saúde.

A comparação entre as causas básicas de óbito das DO identificadas neste estudo e as causas básicas determinadas pela autópsia verbal, considerando-se a DO, neste caso, como padrão ouro, evidenciaram que houve uma boa concordância estatística entre os dois instrumentos.

A concordância estatística entre a informação dos agentes de saúde sobre a causa básica dos óbitos e a revisão da autópsia verbal mostra que os agentes de saúde são capazes de identificar bem um óbito cuja causa básica tenha sido diarréia, porém identificam mal os casos de IRA e sabem diferenciar regularmente outras causas. De um modo geral, é compreensível que os agentes de saúde tenham dificuldade para identificar os casos de IRA, visto que, mesmo em estudos que utilizaram autópsia verbal aplicada por pessoal de nível médio ou superior e revisada por um médico, a sensibilidade e a especificidade para IRA foram regulares ou fracas $(10,17)$. Conforme a orientação que os agentes de saúde recebem, uma das formas de identificar a pneumonia é a presença de respiração rápida. Portanto, é compreensível que estes agentes confundam a dificuldade respiratória própria da prematuridade ou da cardiopatia congênita, ou a acidose metabólica conseqüente à desidratação, como sendo pneumonia. Seria importante, ao treinar os agentes de saúde para reconhecer IRA, chamar sua atenção para a necessária presença de tosse em casos de pneumonia.

É interessante verificar que entre os casos que os agentes de saúde identifi- 
caram como tendo causas básicas distintas de diarréia ou IRA, a prematuridade foi a causa mais freqüentemente identificada pela autópsia verbal, seguida por diarréia, causas não definidas e malformações congênitas. Sugere-se que, com a atual realidade epidemiológica da saúde infantil no Estado, os agentes de saúde sejam orientados para notificar também, separadamente, os casos de prematuridade e malformações congênitas que não sejam de difícil identificação.

A grande maioria dos óbitos não notificados pelos agentes de saúde ocorreram em Quixadá (42 óbitos). Naquele município, a cobertura do programa de agentes de saúde em 1993 e 1994 esteve em torno de 70\%. Quarenta e dois óbitos representam $29 \%$ do total de 144 óbitos identificados em Quixadá; portanto, a notificação de óbitos correspondeu à cobertura de agentes de saúde do município. É importante lembrar que, nos municípios do estudo, o funcionamento do SIPAS pode ter sido melhor do que na média dos outros municípios do Estado, pela ênfase que foi dada ao problema da mortalidade infantil no período. Mesmo com esta observação, os resultados demonstram o potencial deste subsistema de informação.

O CMI dos municípios estudados foi, no caso de Quixadá e Icapuí, duas vezes menor, e no caso de Jucás, 30\% menor do que a taxa obtida no Estado do Ceará no mesmo período, considerando como referência as informações do SIPAS. Como não houve melhorias consideráveis nas condições socioeconômicas das populações estudadas, isto leva a crer que esses coeficientes menores sejam, em parte, resultado das ações primárias de saúde desenvolvidas nos três municípios.

Embora a investigação sobre o itinerário terapêutico das crianças investigadas tenha sido relativamente simplificada, considerando a complexidade desta questão, alguns aspectos podem ser discutidos a partir dos resultados. Observou-se que a grande maioria das famílias procuraram os serviços oficiais de saúde em algum momento da doença que ocasionou o óbito. Nestas famílias, mais de um terço das crianças vieram a falecer posteriormente em casa. Este resultado sugere a ocorrência de fenômenos que merecem intervenção urgente: primeiro, é possível que os serviços de saúde estejam pouco preparados para detectar crianças com doenças graves e potencialmente fatais, perdendo as oportunidades de reverter desfechos negativos; segundo, após internamento por doenças graves, as crianças podem estar recebendo alta hospitalar ainda muito fragilizadas, vindo a falecer posteriormente em casa; terceiro, as crianças levadas aos serviços de saúde e que recebem recomendação para tratamento no domicílio podem ter sua doença agravada e a família pode não estar suficientemente esclarecida quanto à necessidade de retornar aos serviços caso ocorra piora ou quando há sinais de perigo ou de gravidade; quarto, uma criança que adoece por uma doença comum, como diarréia ou resfriado, pode ter seu quadro agravado e a família só procura os serviços de saúde muito tardiamente.

As intervenções necessárias para reverter este quadro certamente terão que envolver uma melhor capacitação das equipes de saúde em atividade no interior do estado para o atendimento a doenças prevalentes na infância (28). Além disso, são necessárias campanhas educativas sobre os sinais de gravidade ou perigo de uma doença em lactentes tanto para a população em geral quanto especificamente para a população de mais baixa renda.

Outro aspecto importante é a grande freqüência com que as famílias recorreram à rezadeira. Embora nos últimos anos agentes de saúde tenham sido implantados em todo o Estado e mesmo tendo havido um aumento na cobertura dos serviços de saúde em geral, a rezadeira, agente não formal de saúde, continua tendo um papel importante na saúde infantil, principalmente para a população de baixa renda. Estes resultados confirmam achados de estudos antropológicos realizados no Ceará (6). Tentar ganhar a cumplicidade deste ator social para as ações básicas de saúde da criança deveria ser colocado na ordem do dia dos sistemas locais de saúde no Ceará.
O fato de os agentes de saúde só terem sido mencionados por menos de um terço das famílias estudadas, mesmo tendo notificado a maioria dos óbitos, alerta para a possibilidade de estes profissionais terem muitas vezes desempenhado o papel de meros notificadores do óbito infantil, quando deveriam ter tido uma maior participação na assistência à criança durante a doença. Também fica clara a necessidade de investir mais na capacitação e na supervisão dos agentes de saúde que, com a implantação do PSF, po-dem se tornar mais efetivos.

Os municípios do Ceará, que já contam hoje com o SIPAS funcionando, podem facilmente criar um bom sistema de vigilância de óbitos infantis, adicionando um instrumento de autópsia verbal à sua rotina. Todos os óbitos infantis poderiam ser monitorados pelo supervisor dos agentes de saúde, através da coleção sistemática das DO junto a hospitais e cartórios e das notificações dos óbitos pelos agentes de saúde. Os óbitos infantis identificados poderiam ser investigados pela autópsia verbal, sendo que as entrevistas poderiam ser executadas pelos enfermeiros supervisores do programa de agentes de saúde ou por um membro da equipe de saúde da família, e revisadas por um profissional médico do município, de preferência pediatra.

A incorporação da técnica de autópsia verbal à prática dos serviços de atenção primária de saúde, leia-se ao cotidiano das equipes de saúde da família, não com o objetivo de saber precisamente a "causa básica biológica" do óbito, mas para estudar a rede de múltiplas causas associadas a cada morte, seria muito útil para que novas mortes fossem evitadas. O ritual de entrevistar uma família em que houve um óbito infantil acaba por sensibilizar as equipes de saúde para esta questão. Estas entrevistas, se discutidas em sessões técnicas com toda a equipe de saúde local, forneceriam elementos para a análise e o planejamento das ações de saúde, tornando tais ações mais efetivas no combate à mortalidade infantil. 


\section{REFERÊNCIAS}

1. Barrêto ICHC, Andrade LOM, De Negri, AA. Nascimento, óbito e cidadania. In: Andrade LOM, Goya N, eds. Sistemas locais de saúde em municípios de pequeno porte: a resposta de Icapuí. Fortaleza: Expressão; 1992. p. 91-142.

2. Benício MHD, Cesar CGL, Gouveia NC. Perfil de morbidade e padrão de utilização de serviços de saúde das crianças brasileiras menores de cinco anos - 1989. In: Fundação Instituto Brasileiro de Geografia e Estatística. Perfil estatístico de crianças e mães no Brasil. Rio de Janeiro: IBGE/UNICEF/INAN; 1992. p. $79-96$

3. Barros FC, Victora CG. Epidemiologia da saúde infantil: um manual para diagnósticos comunitários. 2a ed. São Paulo: Hucitec/ Unicef; 1994.

4. Ceará, Comissão Intersetorial de Coordenação de Ações Relacionadas com a Criança. A saúde das crianças cearenses. Fortaleza: UECE; 1988

5. MacAuliffe JF, Correa LL, Grangeiro GP. Terceira pesquisa de saúde materno-infantil do Ceará - 1994. Fortaleza: Secretaria da Saúde do Ceará, 1995.

6. Silva AC. Viva criança: os caminhos da sobrevivência infantil no Ceará. Fortaleza: Edições Fundação Demócrito Rocha; 1999.

7. Nations MK, Rebhun LA. Angels with wet wings won't fly; maternal sentiment in Brazil and the image of neglect. Cult Med Psychiatry 1988;12(2):141-200.

8. Programa Saúde da Família, saúde dentro de casa/Fundação Nacional de Saúde. Programa de Saúde da Família. Brasília: Ministério da Saúde; 1994.

9. Dal Poz MR, Viana ADV. Reforma em saúde no Brasil: programa de saúde da família, informe final [relatório de pesquisa]. Série Estudos em Saúde Coletiva 166. Rio de Janeiro: Instituto de Medicina Social da Universidade Estadual do Rio de Janeiro; 1998
10. Snow RW, Armstrong JR, Forster D, Winstanley MT, Marsh VM, Newton CF, et al. Childhood deaths in Africa: uses and limitations of verbal autopsies. Lancet 1992;340(8815): $351-355$

11. Mirza NM, Macharia WM, Wafula EM, Aguanda RO, Onyango FG. Verbal autopsy: a tool for determining cause of death in a community. East Afr Med J 1990;67(10):693-699.

12. Victora CG, Smith PG, Vaughan JP, Nobre LC, Lombardi C, Teixeira AM, et al. Evidence of protection by breast feeding against infant deaths from infectious diseases in Brazil. Lancet 1987;2(8554):319-321.

13. Chen LC, Rahman M, Sarder AM. Epidemiology and causes of death among children in a rural area of Bangladesh. Int J Epidemiol 1980; 9(1):25-33.

14. Greenwood BM, Greenwood AM, Bradley AK, Tulloch S, Hayes R, Oldfield FS. Deaths in infancy and early childhood in a wellvaccinated, rural, west African population. Ann Trop Paediatr 1987;7(2):91-99

15. Bang AT, Bang RA, Tale O, Sontakk P, Solanki $\mathrm{J}$, Wargantimar R, et al. Reduction in pneumonia mortality and total childhood mortality by means of community-based intervention trial in Gadchiroli, India. Lancet 1990;336 (8709):201-206.

16. Kalter HD, Gray RH, Black RE, Gultiano A. Validation of postmortem interviews to ascertain selected causes of death in children. Int J Epidemiol 1990;19(2):380-386.

17. Martínez $H$, Reyes $H$, Tomé $P$, Guiscapré $H$, Gutiérrez G. La autopsia verbal: una herramienta para el estudio de la mortalidad en niños. Bol Med Hosp Infant Mex 1993; 50(1):57-63.

18. Ceará, Instituto de Planejamento do Estado do Ceará. Indicadores sociais dos municípios do Ceará. Volume I. Fortaleza: PLANCE/ UNICEF; 1995.
19. Ceará, Instituto de Planejamento do Estado do Ceará. Indicadores sociais dos municípios do Ceará. Volume II. Fortaleza: IPLANCE/ UNICEF; 1995

20. Ceará, Instituto de Planejamento do Estado do Ceará. Indicadores sociais dos municípios do Ceará. Volume III. Fortaleza: IPLANCE/ UNICEF; 1995.

21. Ceará, Instituto de Planejamento do Estado do Ceará.: Indicadores sociais dos municípios do Ceará. Volume IV. Fortaleza: IPLANCE/ UNICEF; 1995.

22. Puffer RR, Serrano CV. Patterns of mortality in childhood: report of the Inter-American Investigation of Mortality in Childhood. Washington, DC: Pan American Health Organization; 1973. (Scientific publication 262).

23. Leão E, Filho PPF. Desnutrição. In: Leão $E$ Viana MB, Correa JE. Pediatria ambulatorial. Belo Horizonte: Coopmed Editora, 1989. p. $155-161$.

24. Pereira MG. Epidemiologia teoria e prática. Rio de Janeiro: Guanabara Koogan; 1995.

25. Brasil. Mortalidade Brasil, 94. Brasília: CENEPI/Fundação Nacional de Saúde: 1997.

26. Hartz ZMA, Champagne F, Leal MC, Contandriopoulos AP. Mortalidade infantil evitável em duas cidades do Nordeste do Brasil: indicador de qualidade do sistema local de saúde. Rev Saude Publ 1996;30(4):310-318.

27. Victora CG, Barros CB. Epidemiologia da desigualdade: um estudo longitudinal de 6000 crianças brasileiras. São Paulo: Hucitec, 1988.

28. Organização Pan-Americana da Saúde. Atenção integrada às doenças prevalentes na infância: introdução. Brasília: PAHO; 1997.

Manuscrito recebido em 14 de junho de 1999. Aceito em versão revisada em 15 de dezembro de 1999.
ABSTRACT

\section{Surveillance of infant deaths in local health systems: assessment of verbal autopsies and of information collected by community health agents}

Knowing the vital statistics of a population is fundamental in controlling morbidity and mortality and improving living conditions. In Brazil, however, the available health information systems do not provide reliable vital statistics. This study was carried out in Quixadá, Icapuí, and Jucás, three municipalities in the state of Ceará that had good coverage by primary health care services. The study used an epidemiological instrument known as a "verbal autopsy" and investigated 215 (90\%) of the 237 deaths of children younger than 1 year identified in 1993 and 1994 in the three communities. We investigated socioeconomic characteristics; sanitary conditions; nutritional status; the course of illness, health care, and death; the cause of death; and the operation of the national mortality information system and of the community health agents system. According to the verbal autopsies, diarrhea was the cause of death in $39 \%$ of the cases, followed by premature birth $(17 \%)$, and acute respiratory infections $(10 \%)$. Even though $79 \%$ of the families had sought formal health care services during the child's illness, $49 \%$ of the infants had died at home. This suggests limited effectiveness in the identification and treatment of sick infants. In $84 \%$ of the cases the family sought help from folk healers. Although community health agents reported $78 \%$ of the deaths, only $29 \%$ of the families had sought help from the agents during the children's illnesses. In terms of the statistical agreement between the information on the cause of death provided by community health agents and by the verbal autopsies, the agreement was good for diarrhea, intermediate for other causes, and low for acute respiratory infections. Making verbal autopsy a routine part of primary health care services in Ceará would provide invaluable information for local health care teams and would raise a critical consciousness fostering a reduction in infant mortality. 